\title{
Simultaneous usage of the LHCb HLT farm for Online and Offline processing workflows
}

\author{
Joel Closier ${ }^{1, *}$, Clara Gaspar ${ }^{1}$, Luis Granado Cardoso ${ }^{1, * *}$, Christophe Haen ${ }^{1, * * *}$, Beat Jost ${ }^{1}$ \\ and Niko Neufeld ${ }^{1}$, on behalf of the LHCb Collaboration.
}

${ }^{1}$ CERN, European Organization for Nuclear Research, Geneva, Switzerland

\begin{abstract}
LHCb}$ is one of the $4 \mathrm{LHC}$ experiments and continues to revolutionise data acquisition and analysis techniques. Already two years ago the concepts of "online" and "offline" analysis were unified: the calibration and alignment processes take place automatically in real time and are used in the triggering process such that Online data are immediately available offline for physics analysis (Turbo analysis), the computing capacity of the HLT farm has been used simultaneously for different workflows : synchronous first level trigger, asynchronous second level trigger, and Monte-Carlo simulation. Thanks to the same software environment, $\mathrm{LHCb}$ can switch seamlessly and fast from Online to Offline workflows, run them simultaneously and thus maximize the usage of the HLT farm computing resources.
\end{abstract}

\section{Introduction}

$\mathrm{LHCb}$ [1] is one of the 4 LHC experiment at CERN and which has been active for 10 years. The data taken by the detector are processed by a set of computers in real time to decrease the amount of information that will be stored on tape for analysis purpose afterwards by physics community.

The computing capacity is quite important and LHCb has decided to use this capacity during idle cycle to process some Monte Carlo generations. In this paper, we will describe the environment of the High Level Trigger (HLT) farm, the various workflows that we use and how we are able to have this concept of "online" and "offline" analysis unified.

\section{HLT farm environment}

The HLT farm is composed of around 1500 PCs, distributed over 60 subfarms. The subfarms are logically divided in the control system and each subfarm row is composed with 24, 28 or 32 PCs each. Each of the subfarms is controlled by a controller PC. These controller nodes

\footnotetext{
* Joel Closier: joel.closier@cern.ch

** Luis Granado Cardoso: luis.granado@cern.ch

**** Christophe Haen: christophe.haen@,cern.ch
} 
are also connected to a top level HLT control node, which manages the availability and allocation of the subfarms for the global Experiment Control System (ECS).

This number of computers represents roughly 12000 cores that are idle when there is no data-taking.

\subsection{Control system ECS}

The Experiment Control System (ECS) [2] in LHCb is based on the SCADA WinCC OA (see next section) with custom LHCb developed components. This application installed on the controller PC managed the HLT tasks on the HLT nodes.

ECS controls the whole experiment as shown in Figure 1 in particular:

- Front end electronics

- High Level Trigger (HLT)

- Data Acquisition System (DAQ)

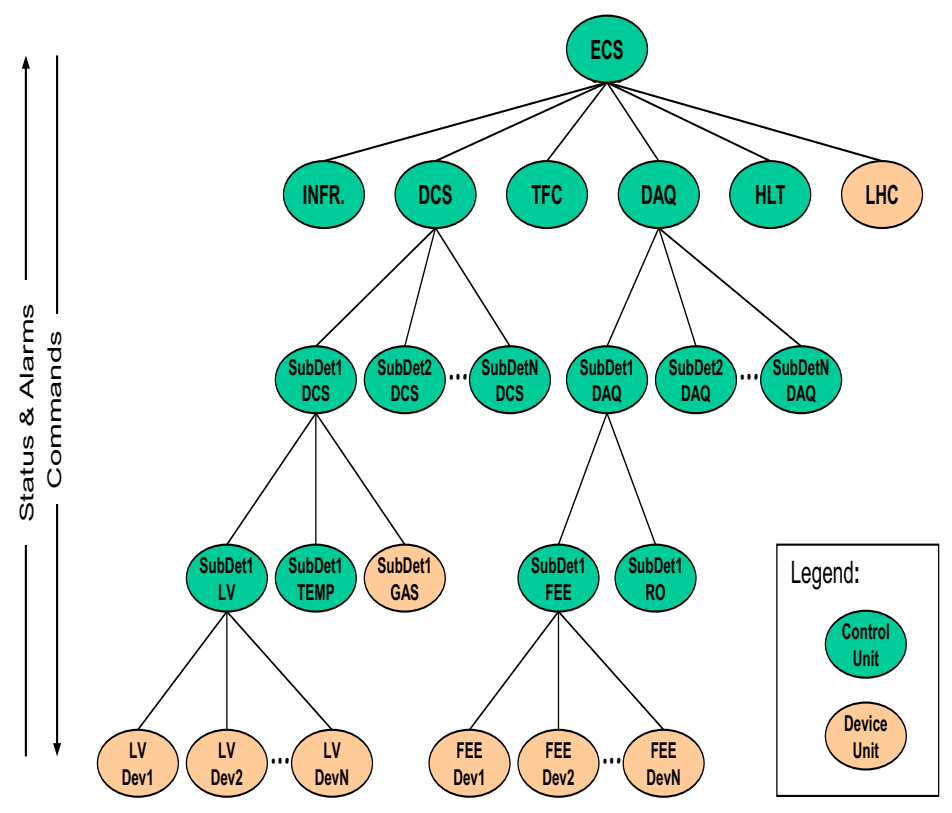

Figure 1. Experiment Control System overview.

ECS is able to configure the whole experiment based on the different states of the LHC accelerator. In the future, we also want to integrate the configuration of the production tools for offline activities (DIRAC tasks) in ECS.

\subsection{WINCC OA}

The SCADA WinCC OA is a commercial product from Siemens. It is used throughout CERN and provides, amongst other things, the user interfaces, the archiving interface and the general alarms for the ECS. 
A control system based on WinCC OA was developed to manage the processing of the offline data on the HLT farm. It is able to monitor and control all the worker nodes on the HLT farm which are organized topographically with farms and subfarms as explained previously.

Each worker node can adopt independent configuration and some of the settings that can be used are:

- Set the exact number of jobs on each machine

- Possibility to set automatically the number of jobs depending on the machine CPU

- In case of automatic configuration, possibility to set the number of cores to be left unused (for DIRAC)

With this configuration, there is no need to change the settings of each node to switch between task and we can easily use just a part of the farm in case some nodes are needed for data taking or for test purposes.

\subsection{DIRAC}

DIRAC [3] (Distributed Infrastructure with Remote Agent Control) INTERWARE (Figure 2) is a software framework for distributed computing providing a complete solution to one (or more) user community requiring access to distributed resources. DIRAC builds a layer between the users and the resources offering a common interface to a number of heterogeneous providers, integrating them in a seamless manner, providing interoperability, at the same time as an optimized, transparent and reliable usage of the resources.

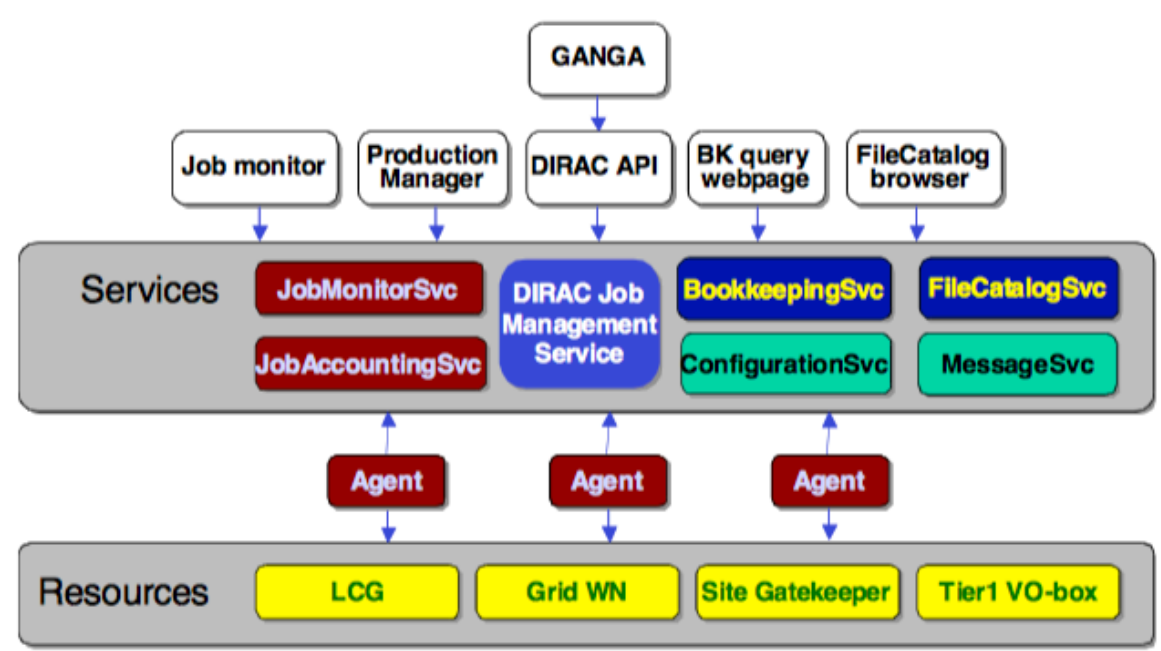

Figure 2. DIRAC Overview of the architecture.

A DIRAC script is a task started on each worker node which perform some actions to run the workflow shown in Figure 3.

- Sets the proper computing environment

- Launches the agent which will also do several actions:

○ Query the DIRAC Workload Management System (WMS) to check if there some task to be executed

If the agent gets a job, it will: 
- Execute in the local disk where the input data, if any, will be downloaded and where the output will be written

- At the end of the task, upload the output to the storage located in the computer centre

- During the execution of the task, information is sent to DIRAC monitoring to follow the progress of the job

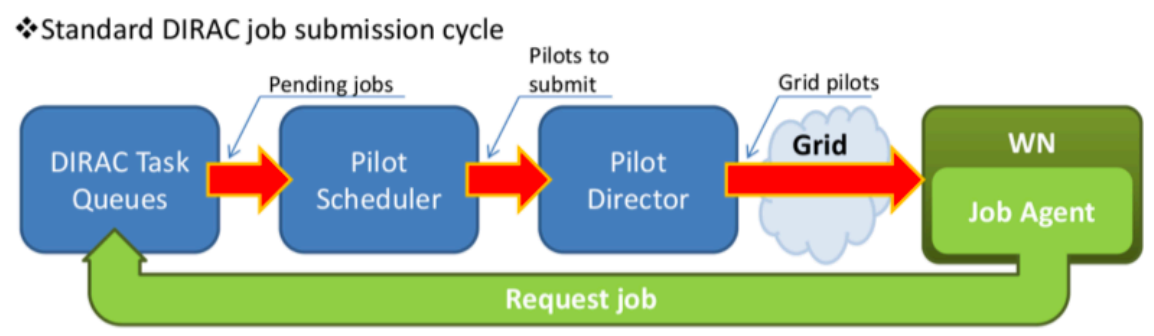

Figure 3. Standard DIRAC job submission cycle.

\subsection{LHCb software environment}

LHCb is using the CernVM File System (CVMFS) to distribute all the LHCb applications. CVMFS is mounted on all the computer centres that are providing computing resources for LHCb: Tier0, Tier1, Tier2 and the HLT.

To run the LHCb applications, we set an environment which is also based on the CernVM File System. The facility to have the same environment eases the initialisation phase of any activities on our computing resources.

\section{LHCb workflows}

In the HLT farm, the resources are used for three main type of activities (Figure 4) that we will describe in the following sections. 


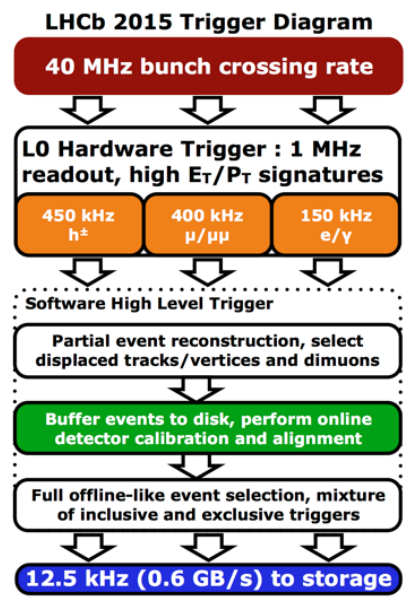

Figure 4. LHCb 2015 Trigger Diagram.

\subsection{High Level Trigger 1}

The first workflow which is used, is the High Level Trigger 1 (HLT1) which reduces the data taking rate from $1 \mathrm{MHz}$ to $100 \mathrm{kHz}$. This workflow is run synchronously in real time.

\subsection{High Level Trigger 2}

The High Level Trigger 2 (HLT2) is run asynchronously on the HLT1 output which has been buffered on the local disk of the HLT farm worker nodes and reduces the rate to $12 \mathrm{kHz}$. This workflow is completely software based and runs on a dedicated computing farm with around 1500 PCs, which represents, roughly 50000 Hyper Core. The HLT2 software is installed on CVMFS on all the worker nodes.

\subsection{Monte Carlo simulation}

The HLT farm is also used during idle cycle to run DIRAC jobs which will simulate events. The simulation software is installed on CVMFS and the environment is set in the same way as the HLT2 software. This eases the usage of the farm for these two different workflows.

\section{HLT farm usage}

The configuration that we have described above, allows LHCb to use the HLT farm resources for various types of workflow and the switch between the two is very easy with the control system that has been developed for this purpose, which controls the configuration of the farm. The Figure 5 show the farm activity of the farm during data taking start-up period. 


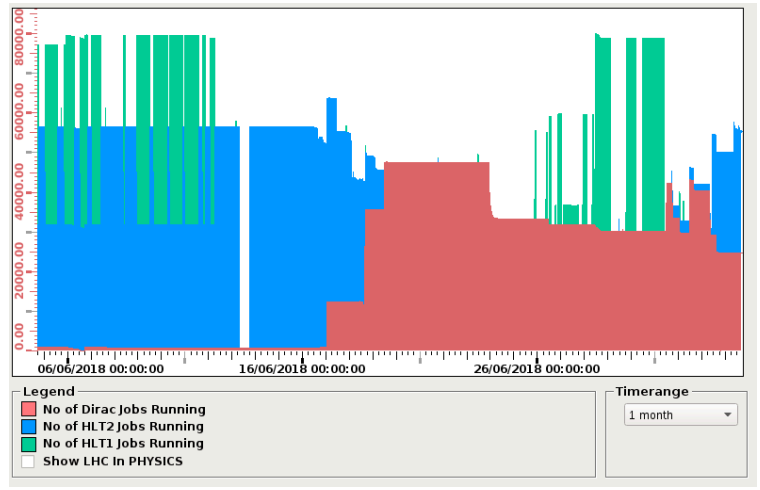

Figure 5. HLT farm activity during data taking start-up period.

The simulation application, based on Gaudi, has been instrumented to catch an interrupt signal. We can see in Figure 6, that when we need to free the HLT farm for data taking, the control system can send an interrupt signal to all the tasks running in the worker nodes and in few minutes, the tasks terminate gracefully without losing any processed events.

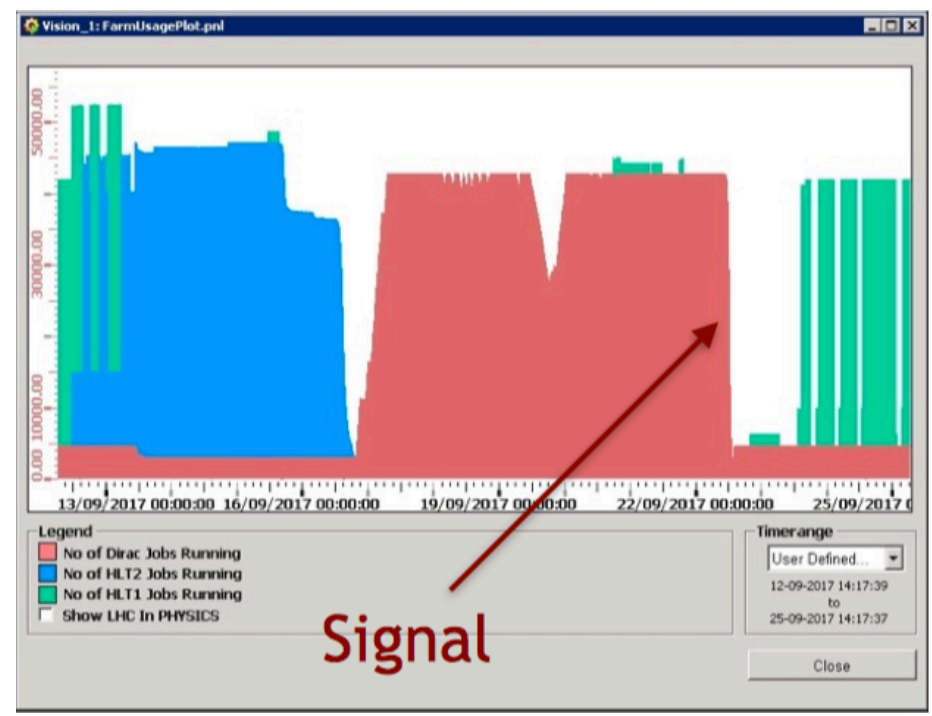

Figure 6. Switch activity in the HLT farm.

The facility to run simulation jobs on the HLT farm provides a big amount of resources to the simulation production. In Figure 7, we can see that CPU cycles used for the simulation production on the HLT farm is bigger than on any other Tier1 used by LHCb in the Grid. In addition, we can see in Figure 8 that the number of running jobs during the period without any data taking put in evidence the benefit of the resources provided by the farm. 


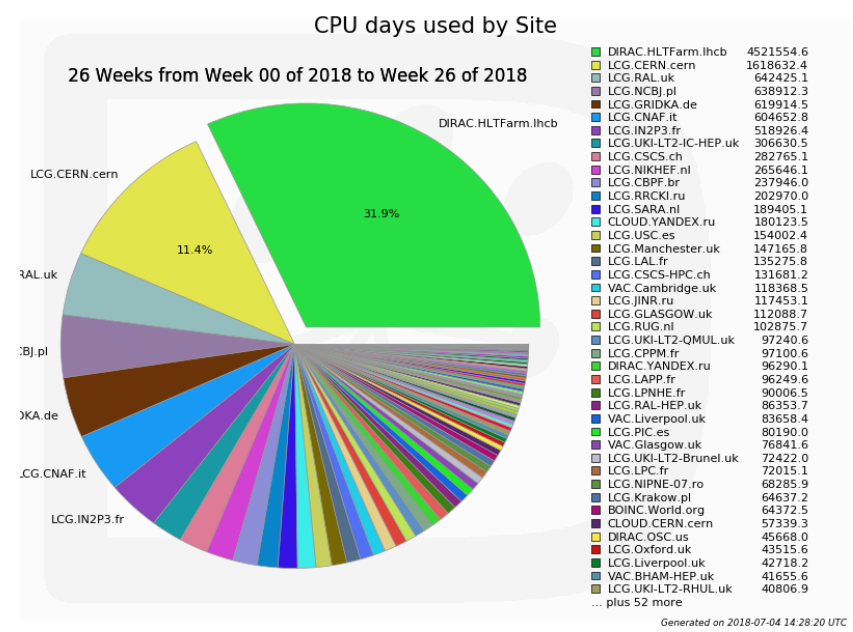

Figure 7. HLT farm CPU days.

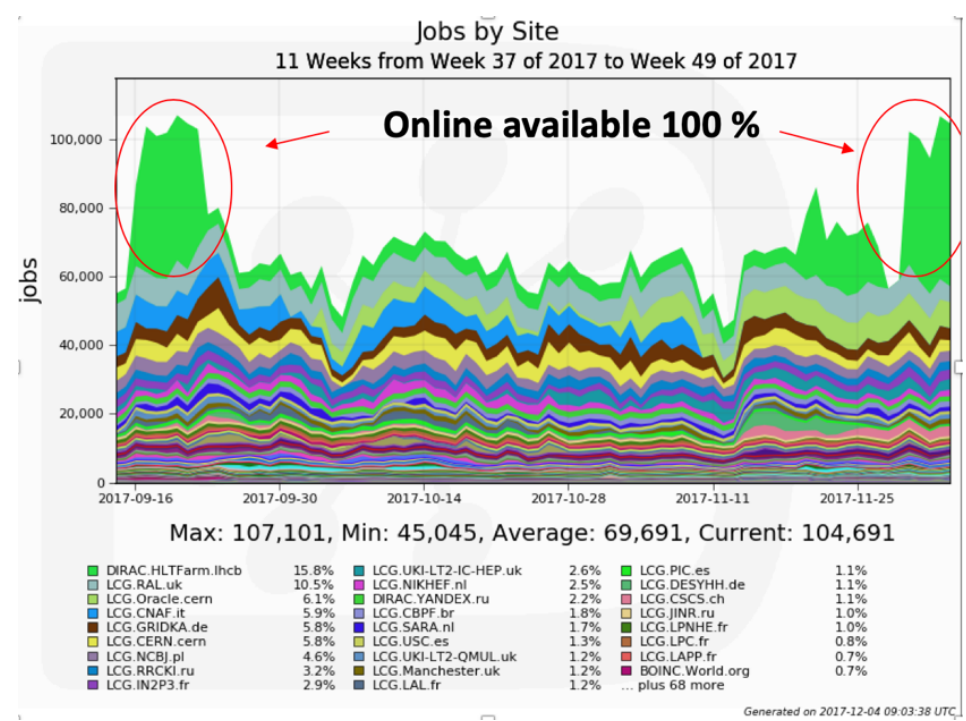

Figure 8. HLT farm usage for 11 weeks.

\section{Conclusion}

The fine grain configuration of the High Level Trigger farm with the control system developed for the usage of the farm for offline tasks, and the usage of CVMFS for the distribution of any LHCb applications, allow the usage of these resources in an optimal way. The usage of the HLT farm is maximized for 2 years and $20 \%$ of the 10 billion events of Monte Carlo that have been produced over the last year, have been done so on the HLT farm. The HLT computing resources are now idle only for maintenance purpose. 


\section{References}

1. The LHCb Collaboration, J. Instrum. 3 S08005 (2008)

2. F Alessio, M C Barandela, O Callot, P-Y Duval, B Franek, M Frank, D Galli, C Gaspar, E v Herwijnen, R Jacobsson, B Jost, N Neufeld, A Sambade, R Schwemmer and P Somogyi, J. Phys.: Conf. Ser. 219, 022009 (2010)

3. A Tsaregorodtsev, J. Phys.: Conf. Ser. 119, 062048 (2008) 